\title{
The NAO Troposphere-Stratosphere Connection
}

\author{
MaArten H. P. Ambaum and Brian J. Hoskins \\ Department of Meteorology, University of Reading, Reading, Berkshire, United Kingdom
}

(Manuscript received 19 October 2001, in final form 18 January 2002)

\begin{abstract}
Using monthly mean data, daily data, and theoretical arguments, relationships between surface pressure variations associated with the North Atlantic Oscillation (NAO), tropopause height, and the strength of the stratospheric vortex are established. An increase in the NAO index leads to a stronger stratospheric vortex, about 4 days later, as a result of increased equatorward refraction of upward-propagating Rossby waves. At tropopause level the effects of the enhanced NAO index and stratospheric polar vortex are opposite, resulting in a lower tropopause over Iceland and a higher tropopause over the Arctic. The raising of the Arctic tropopause leads to a stretching and spinup of the tropospheric column and is therefore associated with a lowering of the surface pressure near the North Pole. For monthly mean data it is found that a standard deviation increase in the NAO index is associated with a $10 \%$ increase in the strength of the stratospheric vortex, as measured by potential vorticity at $500 \mathrm{~K}$. A simple theoretical model predicts that this is associated with about 300-m elevation of the Arctic tropopause, as is observed, and a 5-hPa lowering of the surface pressure at the North Pole. The effects of the spinup of the tropospheric column may project on the NAO pattern so that the stratosphere acts as an integrator of the NAO index.
\end{abstract}

\section{Introduction}

The vertical propagation of Rossby waves was investigated by Charney and Drazin (1961) using linear wave theory. This theory still provides the paradigm for our thinking about the coupling of the stratosphere with the troposphere. For example, in wintertime large-scale horizontal meanders in the tropospheric jet are thought to propagate upward and break at the level of the stratospheric jet. These breaking waves act to decelerate the stratospheric jet. In this way the flow in the troposphere and the stratosphere are connected. Indeed, the strengths of the zonal winds in the stratosphere and troposphere are seen to be significantly correlated. Baldwin et al. (1994) show that the strength of the stratospheric jet is mainly linked with the strength of a pattern in the troposphere very similar to the North Atlantic Oscillation (NAO), which may be viewed as a measure of largescale meanders over the North Atlantic region.

A related observed connection between the stratosphere and the troposphere is that between the total ozone column and Arctic Oscillation (AO), a pattern which is highly correlated with the NAO (e.g., Thompson et al. 2000). It is observed that the change in the total ozone column over the Arctic is negatively correlated with the AO. There is an apparent contradiction here as an increase in AO index is assumed to be associated with an enhanced tropospheric polar jet that is

Corresponding author address: Dr. M. H. P. Ambaum, Dept. of Meteorology, University of Reading, P.O. Box 243, Reading, Berkshire RG6 6BB, United Kingdom.

E-mail: M.H.P.Ambaum@reading.ac.uk normally associated with a depressed tropopause, and consequently a taller stratospheric ozone column.

In the next section we show that the stratospheric component of the NAO plays the crucial role in resolving this paradox. We will establish how the NAO index and the strength of the stratospheric polar vortex, as measured in potential vorticity, are related and how they impact on the height of the tropopause and on the surface pressure. The connections we envision are depicted in a schematic form in Fig. 1. The deeper Icelandic low associated with an enhanced NAO index is associated with a lower tropopause. It is also associated with enhanced equatorward refraction of upward-propagating Rossby waves. Consequently there is less wave breaking in the stratospheric jet, which therefore becomes stronger. The associated generation of a positive potential vorticity anomaly will result in the tropopause over the North Pole rising, which in turn will stretch the tropospheric column below. The relative vorticity thus produced will be associated with a low pressure signal near the North Pole.

\section{Monthly means}

In this section regression maps based on monthly mean winter data (December-March) will be presented. ${ }^{1}$

\footnotetext{
${ }^{1}$ Dr. P. Berrisford is acknowledged for preparing the data as part of the Joint Diagnostics Project. Monthly mean data from an extended ECMWF reanalysis dataset (here comprising years 1979-97) have been used. For the years 1979-95 it is drawn from the European Centre for Medium-Range Weather Forecasts Re-Analysis. In subsequent years routine operational analyses are used.
} 


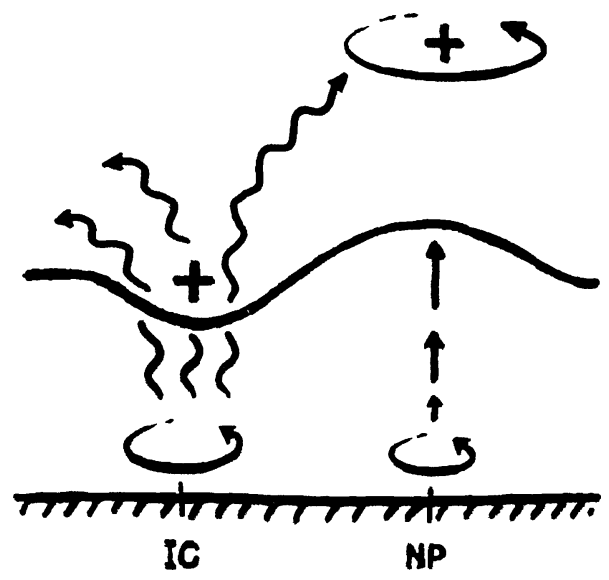

FIG. 1. Schematic of the connections between modulations in the $\mathrm{NAO}$, the height of the tropopause, and the strength of the stratospheric jet. If the NAO index increases, associated with it the cyclonic circulation over Iceland (IC) enhances (circular arrow at IC) and the tropopause (thick solid line) lowers with associated positive potential vorticity anomaly (+); upward-propagating Rossby waves (wavy lines) refract more toward the equator and break less in the stratospheric jet; the stratospheric jet enhances (large circular arrow) with associated positive potential vorticity anomaly $(+)$; the tropopause below this anomaly rises and stretches (vertical arrows) the tropospheric column leading to an enhanced cyclonic circulation over the North Pole (circular arrow at NP).

The wave propagation processes responsible for the connection between the stratosphere and the troposphere are thought to have a timescale of significantly less than a month. Consequently, lagged relationships will appear as instantaneous connections in these regression maps. Processes associated with geostrophic and hydrostatic adjustment also have a timescale of much less than a month. So, for example, tropospheric signatures of stratospheric potential vorticity anomalies will also appear as instantaneous diagnostic connections in these maps.

First we will define some indices that are of relevance in the following. The NAO index (NAO) will be defined as the time series of the first principal component of mean sea level pressure confined over the Atlantic sector. The seasonal cycle is removed by subtracting monthly climatologies. More details can be found in Ambaum et al. (2001). An index measuring the strength of the stratospheric polar vortex (PV500) will be defined as the time series of the first principal component of potential vorticity on the $\theta=500 \mathrm{~K}$ isentropic surface, at approximately $20-\mathrm{km}$ height. The maps of the principal components and graphs of their time series are shown in Fig. 2. These two time series can be used to define a tropospheric NAO index $\left(\mathrm{NAO}_{\mathrm{t}}\right)$ as $\mathrm{NAO}_{\mathrm{t}}=$ NAO - PV500 $\times \operatorname{cov}(\mathrm{NAO}, \mathrm{PV} 500) / \operatorname{cov}(\mathrm{PV} 500$, PV500), which has no linear correlation with the PV500 index $[\operatorname{cov}(a, b)$ is the covariance between $a$ and $b]$. This can be used along with the NAO index to look at features associated with the NAO that are or are not related to the stratospheric component of the NAO. Analogously we can define a stratospheric PV500 index $\left(\mathrm{PV}_{500_{\mathrm{s}}}\right)$ that has no linear correlation with the NAO index. Graphs of these two indices are also in Fig. 2. The linear correlation, $r$, between NAO and PV500 of $r=0.46$ leads to a linear correlation between $\mathrm{NAO}$ and $\mathrm{NAO}_{\mathrm{t}}$ and between PV500 and PV500 ${ }_{\mathrm{s}}$ of $\left(1-r^{2}\right)^{1 / 2}=$ 0.89 .

What are the signatures of these indices on the height of the tropopause? The height of the tropopause was determined using the World Meteorological Organization lapse-rate definition and linear interpolation between data levels for each day. The climatological mean of the height of the tropopause is shown in Fig. 3. Although the direction of the gradient is generally toward the lower latitudes, the picture in the Arctic region is more complicated (Highwood et al. 2000). Minima are located over northern Canada and Japan while over the Arctic the isolines are approximately directed from $60^{\circ} \mathrm{W}$ to $120^{\circ} \mathrm{E}$. In Fig. $3 \mathrm{~b}$ the regression of the height of the tropopause on the NAO index is shown. Many of the features are concentrated around this gra-
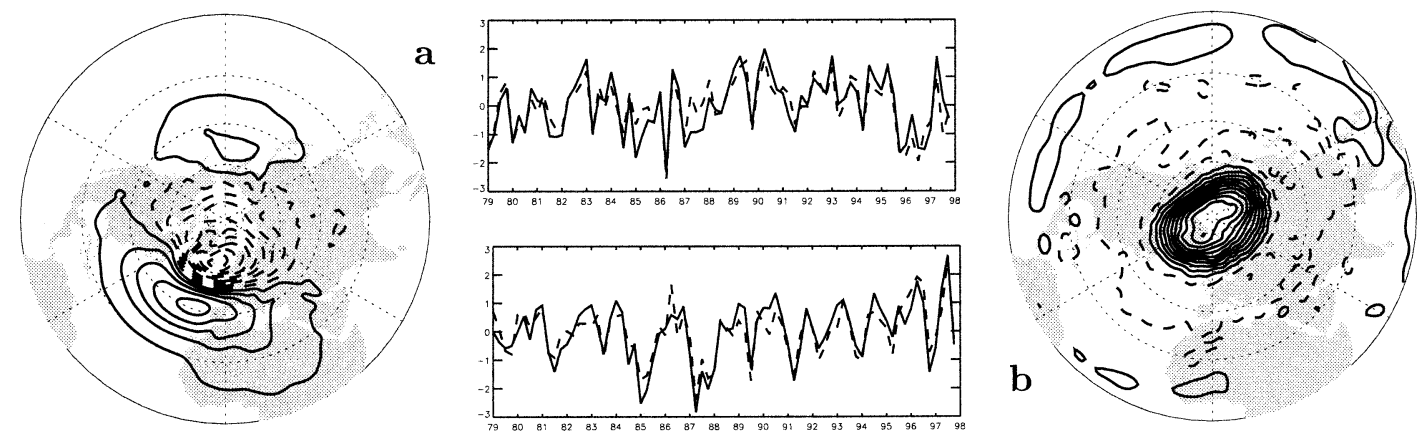

FIG. 2. (a) First principal component of Atlantic sector mean sea level pressure, with contour interval $1 \mathrm{hPa}$ (..., $-0.5,0.5,1.5, \ldots)$. It explains $48 \%$ of the variance over the Atlantic sector and $24 \%$ over the full hemisphere. The solid line in the graph is its standardized time series, the NAO index, and the dashed line is the NAO index. (b) First principal component of the potential vorticity at the $\theta=500 \mathrm{~K}$ isentropic surface, with contour interval 1 PVU. It explains $34 \%$ of the variance. The solid line in the graph is its standardized time series, the PV500 index, and the dashed line is the PV500s index. 

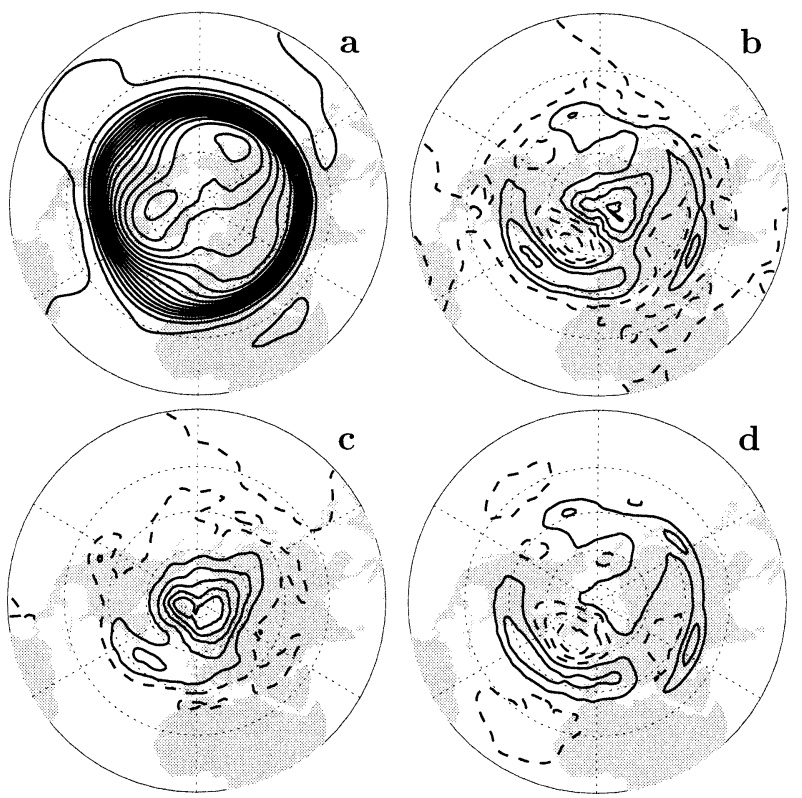

FIG. 3. (a) Dec-Mar climatological mean of tropopause height in meters. The contour interval is $500 \mathrm{~m}$ and the lowest contours over northern Canada and Japan represent $8500 \mathrm{~m}$. (b) Regression of tropopause height with NAO index. The contour interval is $100 \mathrm{~m}$ $(\ldots,-50,50,150, \ldots)$. (c) Same as (b) but for the PV500 index. (d) Same as (b) but for the $\mathrm{NAO}_{\mathrm{t}}$ index.

dient over the Arctic. As expected, the tropopause is higher over the Azores high associated with the NAO, and is lower over the enhanced Iceland low. However, over the rest of the Arctic the tropopause is generally higher.

Key to the higher Arctic tropopause with increased NAO index is the stratosphere. The stratospheric vortex increases in strength with increasing NAO index. The linear correlation between the NAO and PV500 indices of 0.46 is an indication of this as the PV500 index is a good measure of the strength of the stratospheric vortex. The height of the Arctic tropopause increases with the PV500 index, as shown in Fig. 3c. The $\mathrm{NAO}_{t}$ index can be seen as that part of the NAO index that is not linearly associated with increases in the stratospheric vortex strength. In Fig. 3d we see that the regression of tropopause height on this index indeed hardly shows this raised tropopause over the Arctic while the signatures over the Iceland and Azores regions are retained. We conclude that the rising of the tropopause with increasing NAO index is associated with the stratospheric component of the NAO.

The reason why this occurs is the dominance of the stratospheric potential vorticity anomaly. As discussed in Hoskins et al. (1985), isentropic surfaces bend toward isolated positive potential vorticity anomalies-see the schematic in Fig. 4. The tropopause at higher latitudes can be associated with a potential vorticity surface. Similarly, the potential temperature of tropopause parcels will have to be conserved on changes that are not as-

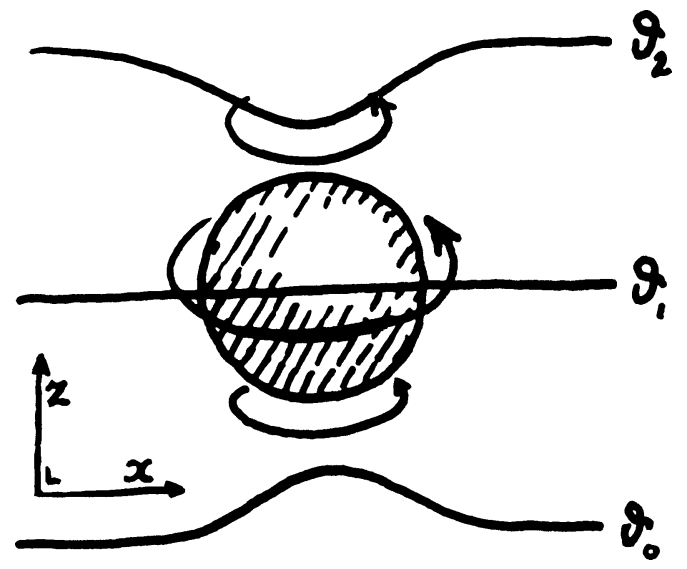

FIG. 4. Schematic of the bending of isentropic surfaces (labeled $\theta_{0}, \theta_{1}$, and $\theta_{2}$ ) toward a positive potential vorticity anomaly. The arrows represent winds associated with the potential vorticity anomaly, becoming weaker away from the anomaly.

sociated with diabatic effects at the tropopause. So we may assume that the potential temperature of the tropopause is more or less fixed for changes in potential vorticity in the stratosphere. This then implies that the tropopause will move upward for a positive stratospheric potential vorticity anomaly.

We can quantify how changes in the height of the tropopause are associated with potential vorticity anomalies in the stratosphere: using isentropic coordinates and hydrostatic balance, the potential vorticity $P$ may be defined as

$$
P=\frac{f+\xi}{\sigma}, \quad \text { with } \sigma=-\frac{1}{g} \frac{\partial p}{\partial \theta}
$$

with the usual notation. A change in potential vorticity $\Delta P$ will be associated with a change in stratification of $\Delta \sigma$ and a change in absolute vorticity of $\Delta \xi$. According to quasigeostrophic scaling, the relative magnitude of the stratification and vorticity contributions is measured by the Burger number $\mathrm{Bu}=(N H / f L)^{2}$. Logarithmic derivatives of the potential vorticity now give

$$
\frac{\Delta P}{P} \approx-(1+\mathrm{Bu}) \frac{\Delta \sigma}{\sigma} .
$$

Unless alternative scales are imposed geometrically, geostrophic adjustment will tend to make the Burger number close to unity for any potential vorticity anomaly, that is, it will tend to make the horizontal length scale $L$ close to the Rossby deformation radius associated with the height scale $H$. We now consider the pressure difference between an isentropic surface above the potential vorticity anomaly $\left(\theta_{\text {top }}\right)$ and the isentropic surface that touches the Arctic tropopause $\left(\theta_{\mathrm{tpp}}\right)$. Finite difference approximations for $\sigma$ and $\Delta \sigma$ in the stratosphere now are

$$
\sigma \approx-\frac{1}{g} \frac{p_{\mathrm{top}}-p_{\mathrm{tpp}}}{\theta_{\mathrm{top}}-\theta_{\mathrm{tpp}}}, \quad \Delta \sigma \approx-\frac{1}{g} \frac{\Delta p_{\mathrm{top}}-\Delta p_{\mathrm{tpp}}}{\theta_{\mathrm{top}}-\theta_{\mathrm{tpp}}},
$$


where it is assumed that $\theta_{\text {top }}$ and $\theta_{\mathrm{tpp}}$ do not change in the process of interest. Now neglecting pressure and its variations at the top of the domain compared to those at the tropopause we find

$$
\frac{\Delta P}{P} \approx-(1+\mathrm{Bu}) \frac{\Delta p_{\mathrm{tpp}}}{p_{\mathrm{tpp}}} .
$$

With a Burger number of about 1 , the fractional change in tropopause pressure is about one-half of, and opposite to, the fractional change of potential vorticity in the stratosphere. The fractional change of potential vorticity in the stratosphere with a positive unit change of the NAO index is about $10 \%$ (not shown), so we conclude that the tropopause pressure decrease due to the stratospheric component of the NAO is about $5 \%$. Using hydrostatic balance this change in tropopause pressure is related to a change in tropopause height as

$$
\Delta h_{\mathrm{tpp}}=-\frac{\Delta p_{\mathrm{tpp}}}{p_{\mathrm{tpp}}} \frac{R T_{\mathrm{tpp}}}{g} .
$$

For a tropopause temperature of about $210 \mathrm{~K}$ this amounts to a change in tropopause height of about 300 $\mathrm{m}$, which is in agreement with the observed increase of tropopause height over the Arctic with NAO (Fig. 3b).

Another example of this quantitative relationship between stratospheric potential vorticity changes and changes in tropopause height is the change of tropopause height with changing PV500 index: in Fig. 2b it is seen that for 1 standard deviation of the PV500 index, the maximum change in the stratospheric potential vorticity values is about 10 PVU. The maximum potential vorticity in the climatological mean is about $60 \mathrm{PVU}$ at that level and it is at the same location as the maximum in Fig. 2b. If the fractional change in potential vorticity of $1 / 6$ is representative for the stratosphere we expect a fractional change in tropopause pressure of about $1 / 12$, which, following the hydrostatic relation, is associated with a change in tropopause height of about $500 \mathrm{~m}$. This is indeed close to the maximum in the regression map of Fig. 3c.

In the above analysis we have ignored the pressure and its variations at the top of the domain because both of them are small compared to the pressure or its variations on the tropopause. This may be viewed as the downward control principle (Haynes et al. 1991) but seen from a different perspective. This principle hinges on the fact that the density decay with height implies a limited capacity above a source region to take up angular momentum while remaining in thermal wind balance. In the downward control setup the forcing associated with the spinup of the stratospheric jet is equilibrated by a secondary circulation below. In our setup the changed stratospheric jet can be thought of as the result of some forcing, while the changed zonal circulation below (see section 4) then is the result of the Coriolis force induced by the secondary circulation associated with that forcing.

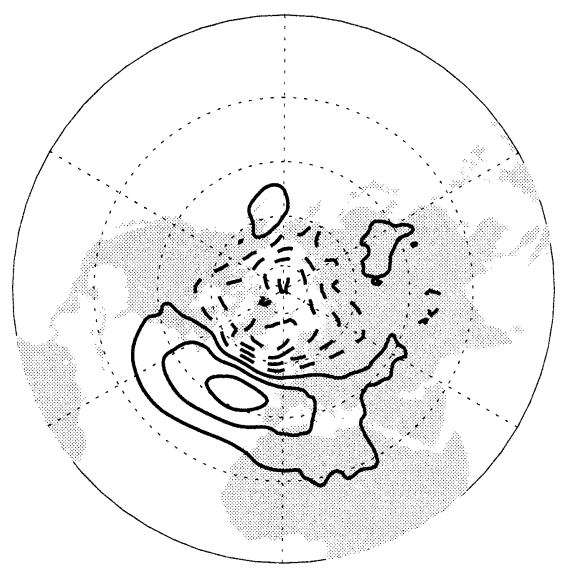

FIG. 5. Regression of mean sea level pressure on the PV500 index. The contour interval is $1 \mathrm{hPa}$, as in Fig. $2 \mathrm{a}$.

Next we will consider how the changes in the troposphere are associated with changes in the stratosphere. It is instructive to see how the mean sea level pressure changes with the PV500 index. Figure 5 shows that changes in the stratospheric vortex strength are mainly associated with mean sea level pressure changes in the Atlantic. Indeed, this pattern is very similar to the NAO pattern in Fig. 2a although the Azores centre of action is displaced northward by about $5^{\circ}$ and the Icelandic centre of action is linked more clearly to one centered almost at the Pole. On regressing the mean sea level pressure on the $\mathrm{PV} 500_{\mathrm{s}}$ index, we find a weaker signature, with extrema of about $\pm 2 \mathrm{hPa}$, which is largely in quadrature with the NAO pattern (not shown). We conclude that the most significant changes in the stratospheric vortex strength are mainly associated with variations in the NAO index.

Baldwin et al. (1994) come to a similar conclusion based on singular value decomposition of 500- and 50$\mathrm{hPa}$ winter mean height fields. They also observe that the strongest connection between these two levels is associated with an NAO-like pattern at the $500-\mathrm{hPa}$ level. They too find some differences in this pattern from what they call the NAO pattern, and most significantly they find that their 500-hPa height SVD pattern also has an Arctic center more extended over the polar cap than the NAO pattern. This feature is confirmed in our analysis, based on a stratospheric index that is not constructed to be connected with the troposphere.

The association of stratospheric vortex strength with mean sea level pressure can be understood within the framework of linear wave propagation. Let us consider the poleward momentum flux by stationary (as defined by monthly means) eddies, $\left[u^{*} v^{*}\right]$, where the square brackets denote a zonal average and the superscripted star the deviation from it. Multiplied by $-r_{0} \cos \phi$, with $r_{0}$ the radius of the earth, this becomes the poleward component of the Eliassen-Palm flux (Edmon et al. 1980). The field $u^{*} v^{*}$ will show regions that contribute most to the zonal mean $\left[u^{*} v^{*}\right]$. However, care must be 

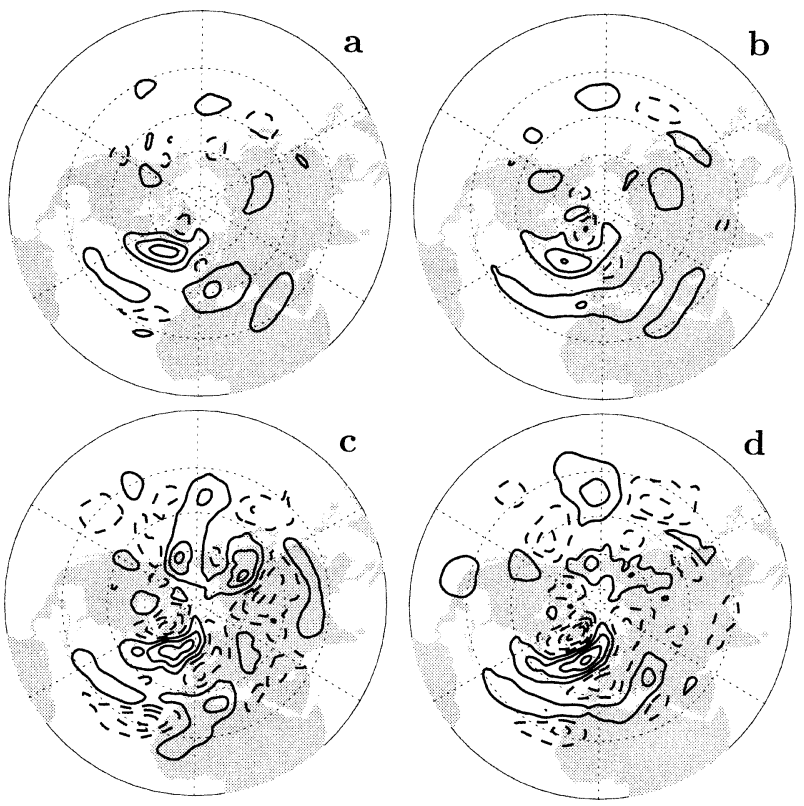

FIG. 6. (a) Regression of $u^{*} v^{*}$ at $300 \mathrm{hPa}$ on the PV500 index. The contour interval is $20 \mathrm{~m}^{2} \mathrm{~s}^{-2}(\ldots,-10,10,30, \ldots)$. (b) Same as (a) but for the $\mathrm{NAO}_{\mathrm{t}}$ index. (c) Regression of $v^{*} T^{*}$ at $300 \mathrm{hPa}$ on the PV500 index. The contour interval is $2 \mathrm{~K} \mathrm{~ms}^{-1}$. (d) Same at (c) but for the $\mathrm{NAO}_{\mathrm{t}}$ index.

taken in interpreting local details, because these will depend on the behavior of the field along the full latitude circle. The regressions of $u^{*} v^{*}$ at $300 \mathrm{hPa}$ on the PV500 and the $\mathrm{NAO}_{\mathrm{t}}$ index are given in Figs. 6a and 6b. Although the two time series are uncorrelated, the regressed fields are remarkably similar; they both show an the increase in stationary poleward momentum flux (i.e., larger equatorward Eliassen-Palm flux) associated with changes over the Atlantic region. The signature of the $\mathrm{NAO}_{\mathrm{t}}$ index on $u^{*} v^{*}$ is associated with the enhancing and lengthening SW-NE jet over the northern Atlantic. The signature of the PV500 index is perhaps more surprising in its localization over the Atlantic and similarity with the $\mathrm{NAO}_{\mathrm{t}}$ signature.

Regressions of the poleward temperature flux $v^{*} T^{*}$ on the PV500 and NAO indices are shown in Figs. 6c and $6 \mathrm{~d}$. The zonal average of this field is proportional to the upward component of the Eliassen-Palm flux. The change of the poleward temperature flux with the $\mathrm{NAO}_{\mathrm{t}}$ and PV500 indices gives a less localized picture than the poleward momentum flux. It is also less obvious whether the zonal averages, $\left[v^{*} T^{*}\right.$, have a clear sign because the anomalies occur with both signs. Similar considerations were put forward by Hartmann et al. (2000) for the zonal mean. They observe that the total poleward heat flux remains more or less the same for increasing AO index, although there is a shift from zonal wavenumber 1 to zonal wavenumber 2 . However, it is remarkable that there is again a high degree of similarity between the regressions over the North Atlantic region.

Under certain assumptions, the Eliassen-Palm flux may be considered as the flux of Rossby wave activity (Edmon et al. 1980). We will not concentrate on providing extra evidence for the picture of the stratosphere being coupled to the troposphere by vertically propagating Rossby waves. What we show here is the remarkable similarity over the Atlantic region in fields related to linear wave fluxes associated with independent modulations of the stratospheric jet and the surface NAO signal. Insofar the above picture is thought valid, our data lead to the interpretation that an enhanced $\mathrm{NAO}_{t}$ index is associated with changes in the Atlantic such that Rossby waves propagating upward into the stratosphere tend to refract more toward the equator. Consequently there is less weakening of the stratospheric jet caused by the subsequent breaking of upward-propagating Rossby waves. This is associated with an enhancing PV500 index. The present study suggests that these changes are mainly due to processes in the Atlantic, associated with the NAO.

We conclude that in monthly mean data there is a strong connection between the stratosphere and the troposphere. The strength of the stratospheric jet is mainly associated with the strength of the NAO pattern. The connection is consistent with the variation in refraction toward the equator of upward-propagating Rossby waves mainly due to changes in the Atlantic. In terms of perturbations in tropopause height, the tropospheric and stratospheric components of the NAO have opposite signs. The tropopause is lowered over the enhanced Icelandic low, but at the same time heightened over the Arctic in association with the enhanced stratospheric jet. It seems that modulations in tropopause heights over the Atlantic and Arctic regions are indirectly connected via the stratosphere.

\section{Daily fields}

We now define daily NAO and PV500 indices. First a daily climatological mean is defined as the centred 30-day running average meaned over all years. Consequently, the climatological mean for a day in the middle of a month is practically identical to the climatological mean for that month. This daily climatological mean is used to define daily anomalies, which are then projected onto the respective principal component fields of Fig. 2.

The lagged autocorrelations for these two indices are plotted as the dashed (NAO) and dotted (PV500) curves in Fig. 7. Only data where one index time series is in the months December-March are included. This explains why the curves are not exactly symmetric around the zero lag line. The autocorrelation of the daily NAO index drops off much more rapidly than that of the daily PV500 index. Furthermore, it does not drop off uniformly with lag but seems to have a richer behavior on timescales of 10-40 days. From the difference in timescales between the indices one may speculate that the daily PV500 index is dominated by slow quasi-baro- 


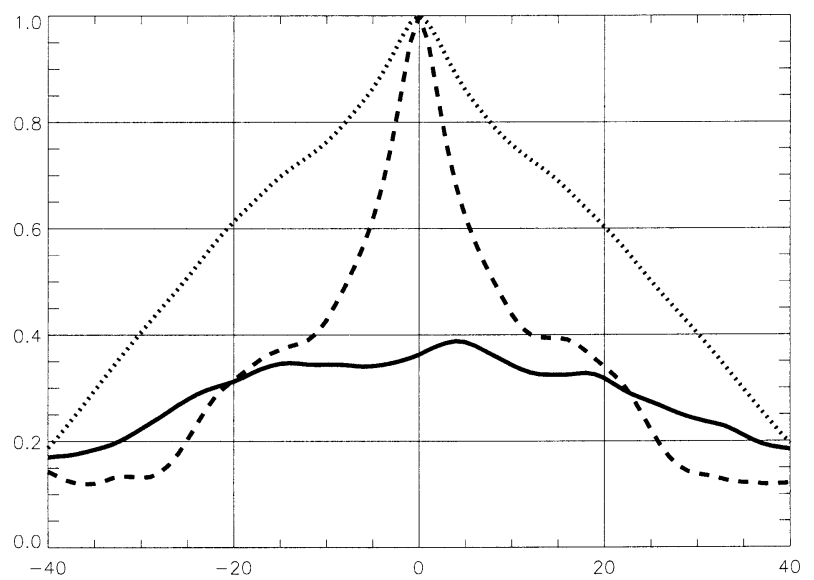

FIG. 7. (solid) Correlation between the daily NAO and PV500 indices as a function of the lag (in days). Positive lag means NAO leading PV500. (dashed) Lagged autocorrelation for the daily NAO index. (dotted) Lagged autocorrelation for the daily PV500 index.

tropic wobbles of the polar vortex, while the daily NAO index on timescales of less than a week is dominated by rapid baroclinic processes in the troposphere associated with weather. However, the "shoulders" in the autocorrelation of the NAO index on timescales of 1015 days are remarkable and perhaps associated with the stratospheric connection.

The solid curve in Fig. 7 shows the lagged correlations between the daily NAO and PV500 indices. Only data where the PV500 index is in the months DecemberMarch are included. There is a small peak of maximum correlation of 0.39 for the NAO index leading the PV500 index by 4 days, which is consistent with a causal relationship where tropospheric anomalies force stratospheric anomalies. This timescale corresponds to the timescale associated with the propagation of a stratospheric wave packet (e.g., Harnik 2002). Randel (1987) observed the same time lag for the Southern Hemisphere wave-1 amplitudes between stratospheric levels and 300 $\mathrm{hPa}$ for the winters of 1983 and 1984. The curve in Fig. 7 does not drop off rapidly with lag because of the long autocorrelation time of the daily PV500 index. This may also explain why the peak at 4 days is only weak.

Figure 8 shows the lagged regressions of daily height of the tropopause on the daily NAO index for lags at 1 -week intervals from -2 to +2 weeks. The general features in these fields are consistent with the connections described in the previous section. The stratospheric signature of the higher Arctic tropopause as seen in Fig. $3 \mathrm{c}$ is always more or less present, because of the long autocorrelation time of the daily PV500 index. The Atlantic centers of Fig. 3d build up toward lag zero and decay after that consistent with the relatively rapid decorrelation of the daily NAO index. Consistent with the lagged relationship between the stratosphere and the troposphere there is an asymmetry in the stratospheric signature in the height of the tropopause that is not present in the tropospheric signature. This can be seen by com-

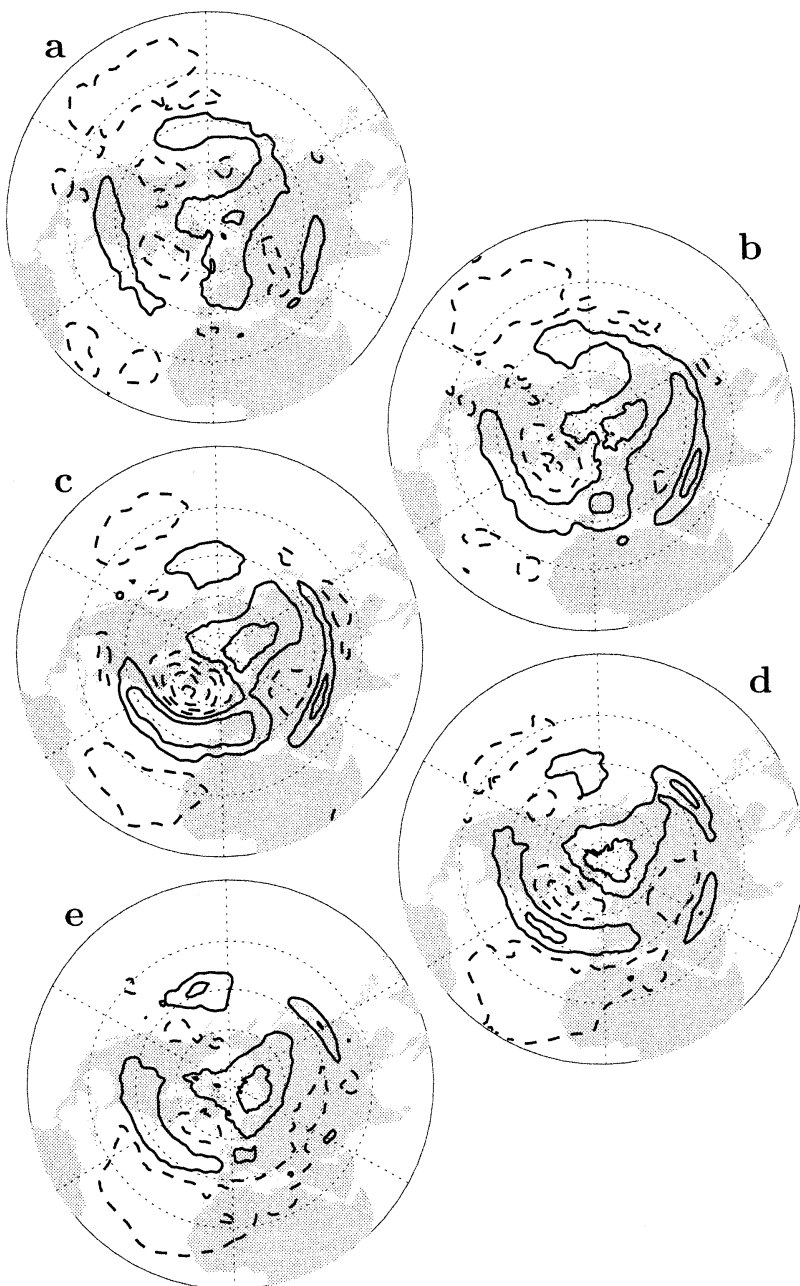

FIG. 8. Lagged regressions of height on the tropopause on the daily NAO index. The lag difference between each image is 1 week, with the central image at zero lag. The contour interval is $100 \mathrm{~m}$, as in Figs. $3 b-3 d$.

paring both Figs. $8 \mathrm{a}(-2$ weeks $)$ and $8 \mathrm{e}(+2$ weeks $)$, and Figs. $8 \mathrm{~b}(-1$ week) and $8 \mathrm{~d}(+1$ week), where the stratospheric signature of the higher Arctic tropopause is stronger for positive lags.

The zero lag regression of the mean sea level pressure on the daily PV500 index is given in Fig. 9b. The similarity to the regression map using monthly mean data (Fig. 5) is clear and expected. However there are some interesting differences such as the relative prominence of the negative Arctic center compared with the negative Icelandic center. The former is most prominent for zero lag. The zero lag regression of tropopause height on the daily PV500 index given in Fig. 9a is also very similar to the monthly mean version (Fig. 3c), but with less sign of an Icelandic center. The differences between the daily and monthly diagnostics are perhaps consistent with the stratospheric connection being most prominent at zero lag with the PV500 index. Because of the long 
autocorrelation of the PV500 index these differences are only expected to be weak as is indeed the case.

\section{Theoretical framework}

We will now consider, in the context of a quasigeostrophic model, how the Arctic stratospheric potential vorticity-tropopause-surface pressure links may be made and how the magnitudes of these can be quantified. The focus is on the polar region in Fig. 1 with zonal symmetry around the Pole assumed. Given that $\beta=0$ at the Pole and the meridional length scale of interest is much less than the planetary radius, the $\beta$ effect will initially be neglected. Also since the zonal average of $v_{g}$ is zero and since we anticipate that the slopes of the isentropes will be much less than the aspect ratio of the motions, we neglect the meridional advection of buoyancy. Therefore the anelastic vorticity and buoyancy equations will be taken to be

$$
\begin{aligned}
& \frac{\partial \xi}{\partial t}=\frac{f}{\rho} \frac{\partial}{\partial z}(\rho w), \\
& \frac{\partial b}{\partial t}=-N^{2} w,
\end{aligned}
$$

where $\rho=\rho_{0} \exp \left(-z / H_{\rho}\right), b=\partial \phi / \partial z, f \xi=\nabla^{2} \phi$, and $f=$ constant. We will assume that we can approximate $\nabla^{2} \phi$ by $-\phi / L^{2}$ for some meridional scale $L$. A good estimate for $L$ is one-half the horizontal radius of the zonally symmetric feature in the field. As usual it is possible to take the $\mathrm{PV} / b$ or $w / \xi$ approaches, and both will be used here.

In the PV/b approach, eliminating $w$ from Eqs. (2) and (3) gives

$$
\frac{\partial q}{\partial t}=0 ;
$$

that is, $q=$ constant, where

$$
q=f \xi+\frac{f^{2}}{\rho} \frac{\partial}{\partial z}\left(\frac{\rho b}{N^{2}}\right) .
$$

For $N^{2}$ constant we have

$$
\frac{N^{2} q}{f^{2}}=\frac{\partial^{2} \phi}{\partial z^{2}}-\frac{1}{H_{\rho}} \frac{\partial \phi}{\partial z}-\frac{N^{2}}{f^{2} L^{2}} \phi \equiv \mathcal{L} \phi .
$$

Boundary conditions are provided by Eq. (3) that on integrating give

$$
\Delta b=-N^{2} \Delta z
$$

with $\Delta z$ changes in height of the top or bottom boundaries.

For the $w / \xi$ approach the time derivatives are eliminated from Eqs. (2) and (3), which leads to the "omega equation":

$$
\mathcal{L} w=0 .
$$

This can be solved with $w$ specified on the boundaries.

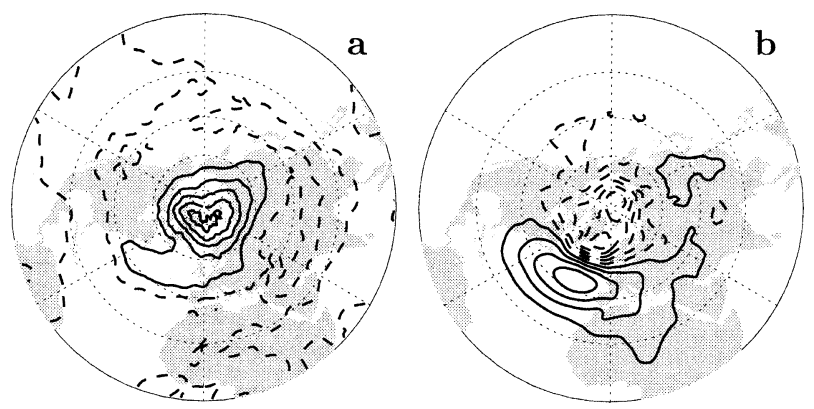

FIG. 9. (a) Regression of tropopause height on the daily PV500 index. The contour interval is $100 \mathrm{~m}$. (b) Regression of mean sea level pressure on the daily PV500 index. The contour interval is 0.5 $\mathrm{hPa}$.

The development in time is then given by the vorticity equation, Eq. (2). The fact the omega equation and the potential vorticity definition involve the same linear operator $\mathcal{L}$ results from the exponential decay of the basicstate density.

Solutions of the potential vorticity equation, Eq. (4), and the omega equation, Eq. (6), both have the form

$$
\begin{aligned}
& e^{z / 2 H_{\rho}}\left(A e^{z / H}+B e^{-z / H}\right), \quad \text { or } \\
& e^{z / 2 H_{\rho}}(C \cosh z / H+D \sinh z / H),
\end{aligned}
$$

where

$$
\frac{1}{H^{2}}=\frac{N^{2}}{f^{2} L^{2}}+\frac{1}{\left(2 H_{\rho}\right)^{2}} .
$$

It is possible to solve a problem with a specific potential vorticity anomaly in the midstratosphere, an interface (the tropopause) between the stratosphere and the troposphere with uniform buoyancy frequencies $N_{s}$ and $N_{t}$, respectively, and a rigid horizontal lower boundary. However, it is more enlightening to consider the two simpler problems associated with the influence down to the tropopause and then from the tropopause to the surface.

It is apparent from the form of Eq. (7a) that the $e$ folding depth in the stratosphere for decay below a potential vorticity anomaly is $\left(1 / H_{s}+1 / 2 H_{\rho}\right)^{-1}$, where $H_{s}$ is determined from Eq. (8) with $N=N_{s}$. Taking values $N_{s}=2 \times 10^{-2} \mathrm{~s}^{-1}, f=1.5 \times 10^{-4} \mathrm{~s}^{-1}, H_{\rho}=6.5 \mathrm{~km}$, and $L=1500 \mathrm{~km}$ (approximately half the radius of the feature in the PV500 map in Fig. 2b) gives $H_{s} \approx 8.5$ $\mathrm{km}$ and a polar stratospheric downward $e$-folding depth of approximately $5 \mathrm{~km}$. This value will vary with the chosen parameters but will always be less than the density scale height $H_{\rho}$. Therefore we can expect an Arctic potential vorticity anomaly in the region of the $500-\mathrm{K}$ surface, at about $20 \mathrm{~km}$, to have a significant impact at the tropopause some $10 \mathrm{~km}$ beneath it. In section 2 above we used a different argument to describe the impact of potential vorticity anomalies on tropopause height. There an inversion is implied by setting $\mathrm{Bu} \approx$ 1 in Eq. (1). The two approaches answer two different 
questions concerning the influence of stratospheric potential vorticity anomalies on the tropopause. The approach in section 2 explains what changes in tropopause height are to be expected given an average potential vorticity anomaly over the depth of the stratosphere. The approach in this section explains how much influence a stratospheric anomaly localized at a certain height will have at tropopause level. Both support the notion that the stratosphere is important for Arctic tropopause height anomalies.

For the tropopause to surface problem we consider a uniform troposphere with a displacement $\Delta h_{\mathrm{tpp}}$ of the tropopause from its basic height $h_{\mathrm{tpp}}$, and first use the $\mathrm{PV} / b$ framework. We use the form of Eq. (7b) with $H_{t}$ determined by Eq. (8) with $N=N_{t}$ and determine $C$ and $D$ by satisfying the boundary conditions $\partial \phi / \partial z=$ 0 at $z=0$, and from Eq. (5), $\partial \phi / \partial z=-N^{2} \Delta h_{\text {tpp }}$ at $z$ $=h_{\mathrm{tpp}}$. For the geopotential height perturbation at the surface this gives

$$
[\phi]_{z=0}=C=-f^{2} L^{2} \frac{\Delta h_{\mathrm{tpp}}}{H_{t}}\left[e^{h_{\mathrm{tpp}} / 2 H_{\rho}} \sinh \left(\frac{h_{\mathrm{tpp}}}{H_{t}}\right)\right]^{-1} .
$$

At the level of the anelastic approximation the associated change in surface pressure is $\Delta p=[\rho \phi]_{z=0}$.

Before determining magnitudes implied by these results, we indicate how the same result can be achieved using the $w / \xi$ framework. Solving Eq. (6) with the boundary conditions $w=0$ at $z=0$ and $w=w_{\text {tpp }}$ at $z$ $=h_{\mathrm{tpp}}$ gives

$$
w=w_{0} e^{z / 2 H_{\rho}} \sinh \left(z / H_{t}\right),
$$

where

$$
w_{0} e^{h_{\mathrm{tpp}} / 2 H_{\rho}} \sinh \left(h_{\mathrm{tpp}} / H_{t}\right)=w_{\mathrm{tpp}} .
$$

Therefore, the vorticity stretching at the surface is

$$
\left[\frac{f}{\rho} \frac{\partial}{\partial z}(\rho w)\right]_{z=0}=\frac{f w_{0}}{H_{t}} .
$$

Time integration of the surface vorticity equation then gives

$$
[\Delta \xi]_{z=0}=f \frac{\Delta h_{\mathrm{tpp}}}{H_{t}}\left[e^{h_{\mathrm{tpp}} / 2 H_{\rho}} \sinh \left(\frac{h_{\mathrm{tpp}}}{H_{t}}\right)\right]^{-1} .
$$

Setting $\Delta \xi=-\phi / f L^{2}=-\Delta p /\left(\rho f L^{2}\right)$ then gives the same result as that from the PV/b approach, Eq. (9). The PV/b approach is perhaps more direct but the $w / \xi$ approach has the advantage of making quantitative the physical picture near the pole sketched in Fig. 1 of the rising tropopause stretching the tropospheric column below.

We now use tropospheric values $N_{t}=10^{-2} \mathrm{~s}^{-1}, f=$ $1.5 \times 10^{-4} \mathrm{~s}^{-1}$, and $H_{\rho}=8 \mathrm{~km}$. The value of $L$ is not clear. Taking it to be the half radius of the tropopause and surface pressure signatures in Figs. $3 \mathrm{c}$ and 5, respectively, suggest $L \approx 1000 \mathrm{~km}$. This gives $H_{t} \approx 11$ $\mathrm{km}$, with relatively small sensitivity of it and therefore of the vorticity perturbation, Eq. (10), to the actual choice of $L$. However, the surface pressure and the geopotential height [Eq. (9)] are much more sensitive, being proportional to $L^{2}$. For $h_{\mathrm{tpp}}=8 \mathrm{~km}$ and $\Delta h_{\mathrm{tpp}}=500$ $\mathrm{m}$, which corresponds to the change in tropopause height associated with 1 standard deviation of the PV500 index for monthly mean data, we find stretching in the troposphere would give $[\Delta \xi]_{z=0} \approx 0.5 \times 10^{-5} \mathrm{~s}^{-1}$, which is close to values of observed regressions of relative vorticity on the PV500 index of about $0.3 \times 10^{-5} \mathrm{~s}^{-1}$ (not shown). With $L=1000 \mathrm{~km}$ and $\rho(0)=1.2 \mathrm{~kg}$ $\mathrm{m}^{-3}$, we obtain $\Delta p=9 \mathrm{hPa}$. This is larger than the observed value of about $3.5 \mathrm{hPa}$ in Fig. 5. However, given the sensitivity to the choice of $L$, a moderate reduction in it would correct this. Also surface friction effects have been omitted in this calculation and would be expected to reduce the magnitude of the pressure perturbation. Similar numbers apply also for the daily data as seen in Figs. 9a and 9b.

The results from the simple quasigeostrophic model used here showing the impact of lower-stratospheric polar potential vorticity anomalies on polar surface pressure are consistent with those given by Hartley et al. (1998) and Black (2002). However, the spherical quasigeostrophic models used in these papers are not consistent with global integral constraints on potential vorticity and vorticity. As yet, we are unsure about the potential effects this may have on the results.

Higher-order models would include the effect in the vorticity and buoyancy equations of advection by the convergent meridional motion associated with the vortex stretching. A $-\beta v$ term on the right-hand side of the vorticity equation, Eq. (2), would act to reduce the impact of vortex stretching away from the Pole and therefore tend to localize the vorticity increase more to the polar region. Or equivalently, material circuits would contract as the relative circulation around them increases. This is perhaps consistent with the observed change in meridional scale from the lower stratosphere to the troposphere (see, e.g., Figs. 9a and 9b). Similarly, the advection of low potential vorticity air and warm air near the tropopause toward the polar region would act to decrease the vorticity increase in a region surrounding the polar cap. This effect could be somewhat reduced by warm air advection near the surface. Calculations with more complete spherical models are required to quantify these effects and treat a sloping tropopause more accurately.

The 300-m rise in the Arctic tropopause associated with 1 standard deviation of the monthly NAO index would according to the calculations be associated with some $5 \mathrm{hPa}$ lowering in Arctic surface pressure due to the connection with the stratosphere, assuming similar horizontal length scales. This number can be compared with the observed amplitude of the NAO pattern at the North Pole of about 3.5 hPa as in Fig. 2a. Given the overestimation by our frictionless calculation this may suggest that perhaps more than half of magnitude of the 


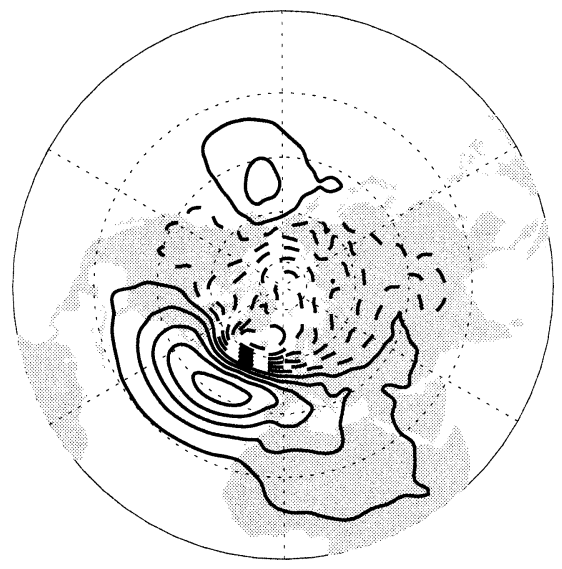

FIG. 10. Regression of mean sea level pressure on the daily NAO index with a positive lag of 1 week, which corresponds to the time of Fig. 8d. The contour interval is $0.5 \mathrm{hPa}$.

NAO pattern at the North Pole is due to its connection with the stratosphere. In Fig. 10 the regression of mean sea level pressure on the daily NAO index is shown with a lag of 1 week (the lag 0 regression map is essentially the same as in Fig. 2a). This image should be compared with the same regression for the tropopause height in Fig. 8d. From both images we can see that a week after an NAO event the stratospheric signature over the Arctic has become more prominent relative to the Atlantic signature. Indeed, the spatial correlation of the daily PV500 regression pattern in Fig. 9b with the lagged daily NAO regression pattern in Fig. 10 is higher than with the pattern in Fig. 2a or the lag 0 regression map. This is consistent with the period after an NAO event being more influenced by its stratospheric component. Our simple model suggests that the observed tropopause rise of about $150 \mathrm{~m}$ over the Arctic in Fig. $8 \mathrm{~d}$ is associated with a surface pressure drop of about $2.5 \mathrm{hPa}$ near the Pole, which is close to the observed pressure signal at the North Pole in Fig. 10.

\section{Conclusions}

The picture of the troposphere-stratosphere connections associated with the NAO as supported in this paper is that given in Fig. 1. The NAO has a stratospheric component that is coupled to its tropospheric component via wave propagation and via geostrophic and hydrostatic adjustment.

Regression maps of monthly mean and daily data indicate that anomalies in the stratospheric vortex strength (as measured by potential vorticity) are associated with anomalies in the Atlantic region, that are very similar to the signatures of the NAO. At uppertropospheric levels we find that linearly independent indices of stratospheric vortex strength and NAO project onto a very similar pattern of anomalous eddy momentum flux localized over the Atlantic region. A high NAO index leads a strong stratospheric vortex with a lag of about four days. The picture emerges of the stratospheric vortex that can only "see" NAO-like variations in the troposphere that are communicated upward by modulating the equatorward refraction of upwardly propagating Rossby waves.

A second aspect to this coupling is the direct effect of stratospheric vortex anomalies on the troposphere through geostrophic and hydrostatic adjustment. A positive stratospheric potential vorticity anomaly is associated with an elevated tropopause around the polar cap. Through stretching, this elevated tropopause leads to enhanced geostrophic vorticity in the troposphere and associated lower pressure near the Pole.

The connection between stratospheric potential vorticity anomalies and the troposphere has been quantified. A very idealized quasigeostrophic model view from the potential vorticity-buoyancy and vertical velocity-vorticity perspectives has supported the physical nature of the hypothesised connections. A significant positive Arctic potential vorticity anomaly at about $20-\mathrm{km}$ altitude can be expected to have a significant raised polar tropopause associated with it. This raised tropopause can be expected to have an associated significant Arctic low pressure anomaly. The polar stratospheric potential vorticity-tropopause height-surface pressure linkages would apply equally to the Southern Hemisphere and in particular to the southern annular mode (e.g., Hartmann et al. 2000).

Our theoretical model predicts that a fractional change in stratospheric potential vorticity is associated with a fractional change in tropopause pressure that is one-half in magnitude and opposite in sign. So a 1 standard deviation increase in the NAO index, which is associated with a $10 \%$ increase in stratospheric potential vorticity, will lead to an associated 5\% drop in Arctic tropopause pressure. This corresponds to a $300-\mathrm{m}$ rise of the Arctic tropopause. These predictions are in accord with the observed regressions. Such a rise of the Arctic tropopause is estimated to lead to a $5-\mathrm{hPa}$ drop in pressure close to the North Pole. This value is somewhat higher than the observed pressure signal perhaps due to the simplicity of the model. In subsequent research it is hoped to add some more complexity to this model to see if the anticipated increase in realism is attained.

The theoretical discussion in this paper and the schematic in Fig. 1 have not tackled the final part of the loop for the Northern Hemisphere. The induced low pressure over the Pole may project directly onto the NAO pattern. However, it is more likely that a torque applied to the Arctic troposphere produces a dynamical response in the NAO "mode." We plan to look at this last link in the NAO Iceland troposphere-Arctic stratosphere-Arctic tropopause-NAO chain.

Because on average the stratosphere reacts with a lag of about four days and because of the slower timescales in the stratosphere, the stratosphere may introduce a memory in the NAO index through the chain of connections described above. An indication of this memory 
may be the "shoulders" in the autocorrelation of the daily NAO index (Fig. 7) at 10-15 days and the very recent results of Thompson and Wallace (2001) and Baldwin and Dunkerton (2001) suggest some predictability in Northern Hemisphere high-latitude weather associated with it.

Acknowledgments. MHPA was funded by the U.K. Natural Environment Research Council through the U.K. Universities Global Atmospheric Modeling Programme and the COAPEC programme.

\section{REFERENCES}

Ambaum, M. H. P., B. J. Hoskins, and D. B. Stephenson, 2001: Arctic Oscillation or North Atlantic Oscillation? J. Climate, 14, 34953507.

Baldwin, M. P., and T. J. Dunkerton, 2001: Stratospheric harbingers of anomalous weather regimes. Science, 294, 581-584.

— between winter-mean tropospheric and stratospheric circulation anomalies. Geophys. Res. Lett., 21, 1141-1144.

Black, R. X., 2002: Stratospheric forcing of surface climate in the Arctic Oscillation. J. Climate, 15, 268-277.

Charney, J. G., and P. G. Drazin, 1961: Propagation of planetary- scale disturbances from the lower into the upper atmosphere. $J$. Geophys. Res., 66, 83-109.

Edmon, H. J., B. J. Hoskins, and M. E. McIntyre, 1980: EliassenPalm cross sections for the troposphere. J. Atmos. Sci., 37, 26002616.

Harnik, N., 2002: The evolution of a stratospheric wave packet. $J$. Atmos. Sci., 59, 202-217.

Hartley, D. E., J. T. Villarin, R. X. Black, and C. A. Davis, 1998: A new perspective on the dynamical link between the stratosphere and troposphere. Nature, 391, 471-474.

Hartmann, D. L., J. M. Wallace, V. Limpasuvan, D. W. J. Thompson, and J. R. Holton, 2000: Can ozone depletion and global warming interact to produce rapid climate change? Proc. Natl. Acad. Sci., 97, 1412-1417.

Haynes, P. H., C. J. Marks, M. E. McIntyre, T. G. Shepherd, and K. P. Shine, 1991: On the "downward control" of extratropical diabatic circulations by eddy-induced mean zonal forces. J. Atmos. Sci., 48, 651-678.

Highwood, E. J., B. J. Hoskins, and P. Berrisford, 2000: Properties of the Arctic tropopause. Quart. J. Roy. Meteor. Soc., 126, 15151532 .

Hoskins, B. J., M. E. McIntyre, and A. W. Robertson, 1985: On the use and significance of isentropic potential vorticity maps. Quart. J. Roy. Meteor. Soc., 111, 877-946.

Randel, W. J., 1987: A study of planetary waves in the southern winter troposphere and stratosphere. J. Atmos. Sci., 44, 917-935.

Thompson, D. W. J., and J. M. Wallace, 2001: Regional climate impacts of the Northern Hemisphere annular mode. Science, 293, $85-89$.

- - , and G. C. Hegerl, 2000: Annular modes in the extratropical circulation. Part II: Trends. J. Climate, 13, 1018-1036. 